Supporting Information

\title{
Low temperature adsorption of hydrogen on nanoporous
}

\section{aluminophosphates: Effect of pore size}

Sung Hwa Jhung, Hye-Kyung Kim, Ji Woong Yoon, and Jong-San Chang ${ }^{*}$

Research Center for Nanocatalysts, Korea Research Institute of Chemical Technology (KRICT), P.O. Box, 107, Yusung, Daejeon 305-600, Korea, jschang@krict.re.kr 
Supporting Table 1. Structures and synthesis conditions of various AlPOs.

\begin{tabular}{|c|c|c|c|c|c|c|}
\hline $\begin{array}{l}\text { Type of } \\
\text { AlPOs }\end{array}$ & $\begin{array}{c}\text { IZA } \\
\text { structure } \\
\text { code } \\
\end{array}$ & MR & $\begin{array}{l}\text { Pore size } \\
(\AA * \AA)\end{array}$ & Reactant composition (molar ratio) & $\begin{array}{c}\text { Synthesis } \\
\text { method and } \\
\text { conditions }\end{array}$ & $\begin{array}{l}\text { Calcination } \\
\text { conditions }\end{array}$ \\
\hline VPI-5 & VFI & 18 & $12.7 * 12.7$ & 1.0 $\mathrm{Pr}_{2} \mathrm{NH}: 1.0 \mathrm{Al}_{2} \mathrm{O}_{3}: 1.0 \mathrm{P}_{2} \mathrm{O}: 100 \mathrm{H}_{2} \mathrm{O}$ & $\begin{array}{c}\mathrm{MW}^{\mathrm{a}} \\
405 \mathrm{~K}, 15 \text { min }\end{array}$ & $573 \mathrm{~K}, 20 \mathrm{~h}^{\mathrm{b}}$ \\
\hline AlPO-5 & AFI & 12 & $7.3 * 7.3$ & 1.2 triethylamine:1.0 $\mathrm{Al}_{2} \mathrm{O}_{3}: 1.0 \mathrm{P}_{2} \mathrm{O}: 50 \mathrm{H}_{2} \mathrm{O}$ & $\begin{array}{c}\mathrm{MW} \\
453 \mathrm{~K}, 30 \mathrm{~min}\end{array}$ & $823 \mathrm{~K}, 6 \mathrm{~h}$ \\
\hline AlPO-11 & AEL & 10 & $6.5 * 4.0$ & $1.0 \mathrm{Pr}_{2} \mathrm{NH}: 1.0 \mathrm{Al}_{2} \mathrm{O}_{3}: 1.0 \mathrm{P}_{2} \mathrm{O}: 100 \mathrm{H}_{2} \mathrm{O}$ & $\begin{array}{c}\text { MW, } \\
453 \mathrm{~K}, 30 \mathrm{~min}\end{array}$ & $823 \mathrm{~K}, 6 \mathrm{~h}$ \\
\hline AlPO-25 & ATV & 8 & $4.9 * 3.0$ & 1.0 pyrrolidine: $1.0 \mathrm{Al}_{2} \mathrm{O}_{3}: 1.0 \mathrm{P}_{2} \mathrm{O}: 100 \mathrm{H}_{2} \mathrm{O}$ & $\begin{array}{c}\mathrm{CE},{ }^{\mathrm{a}} \\
423 \mathrm{~K}, 150 \mathrm{~h}\end{array}$ & $873 \mathrm{~K}, 6 \mathrm{~h}^{\mathrm{C}}$ \\
\hline
\end{tabular}

\footnotetext{
${ }^{\mathrm{a}} \mathrm{MW}$ : microwave irradiation; CE: conventional electric heating

${ }^{\mathrm{b}}$ in-situ calcination and evacuation

${ }^{\mathrm{c}}$ in-situ calcination, evacuation and conversion of AlPO-21 to AlPO-25
} 


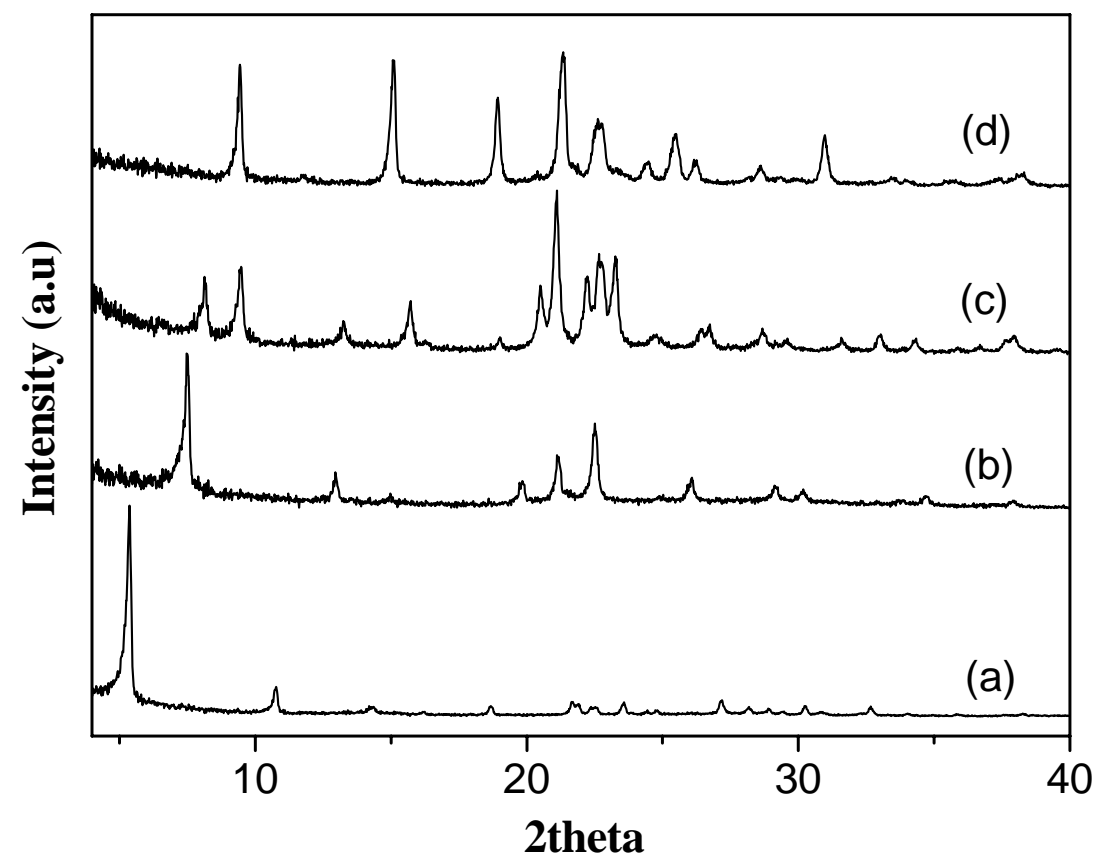

Supporting Figure 1. XRD patterns of various AlPOs: (a) VPI-5, (b) AlPO-5, (c) AlPO-11, and (d) AlPO-25. 


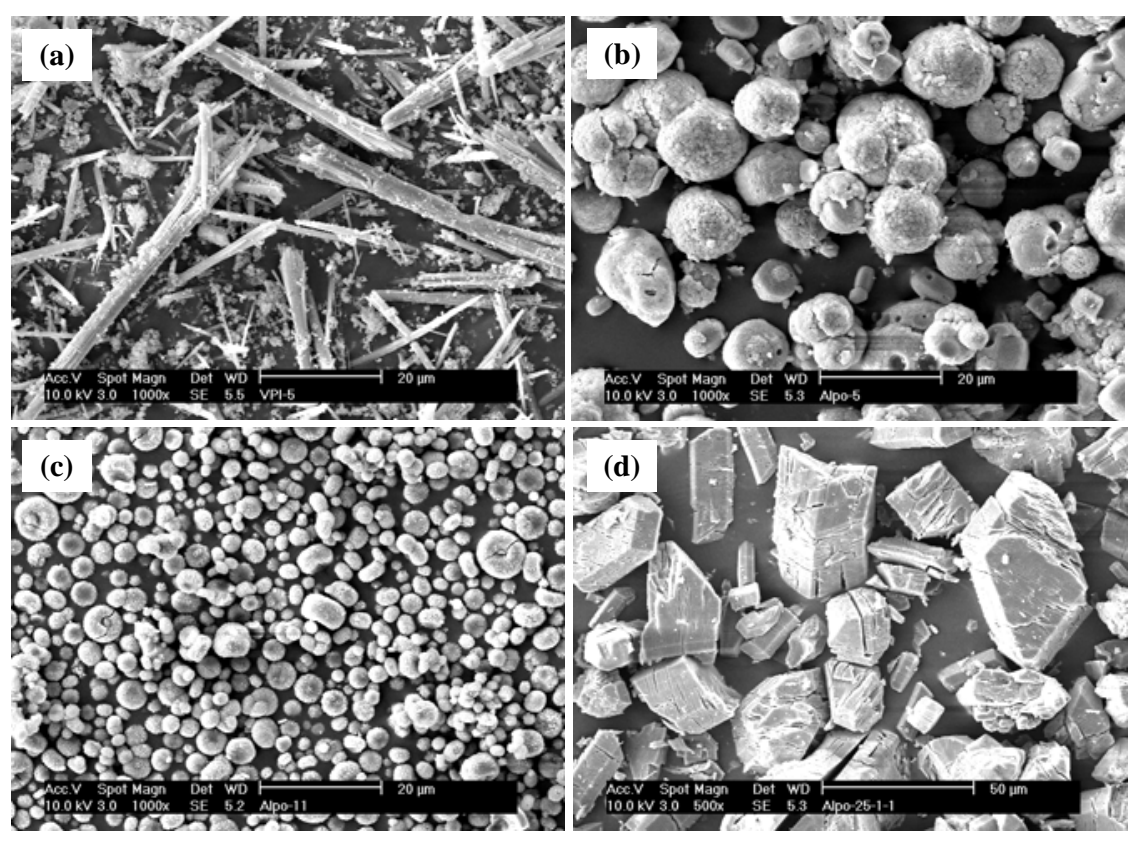

Supporting Figure 2. SEM images of AlPOs before grinding: (a) VPI-5, (b) AlPO-5, (c) AlPO-11, and (d) AlPO-25. 

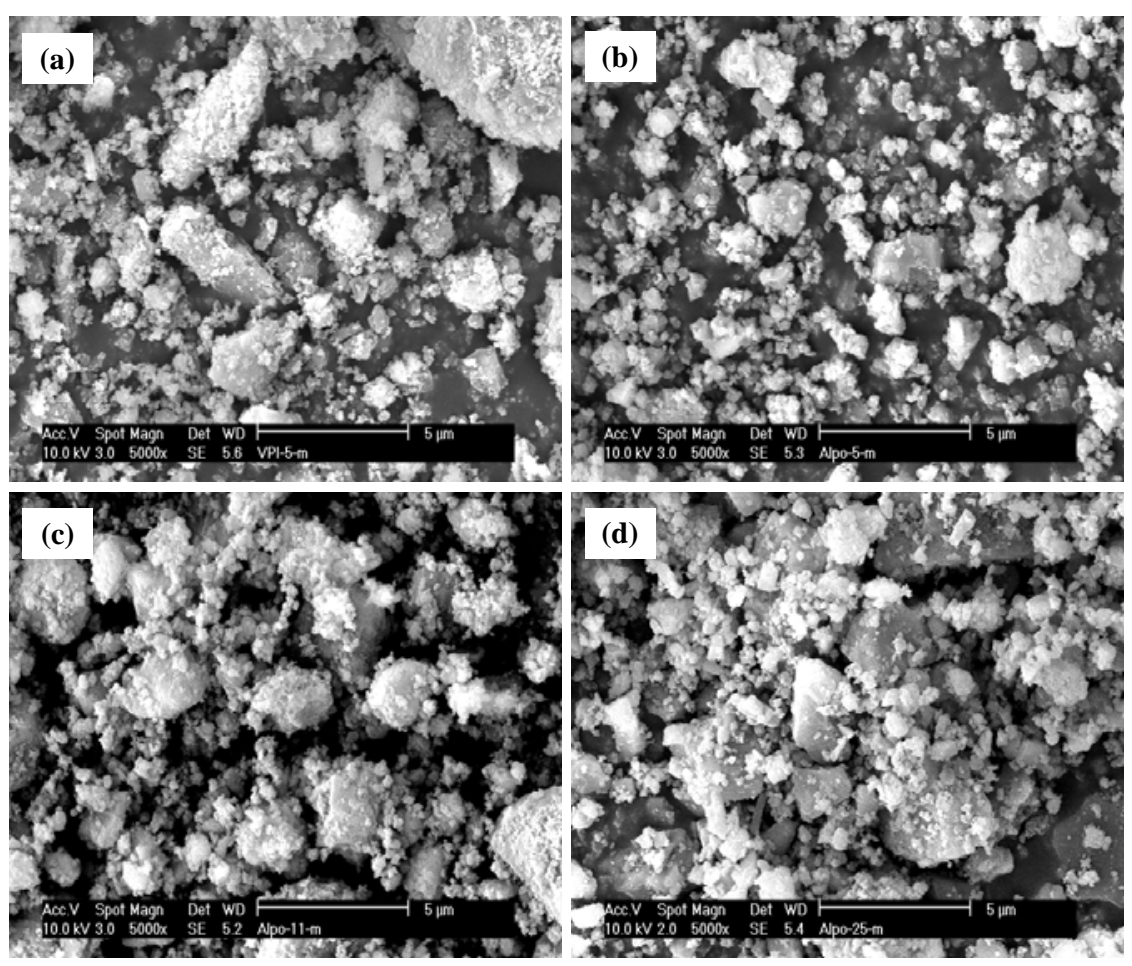

Supporting Figure 3. SEM images of AlPOs after grinding: (a) VPI-5, (b) AlPO-5, (c) AlPO-11, and (d) AlPO-25. 


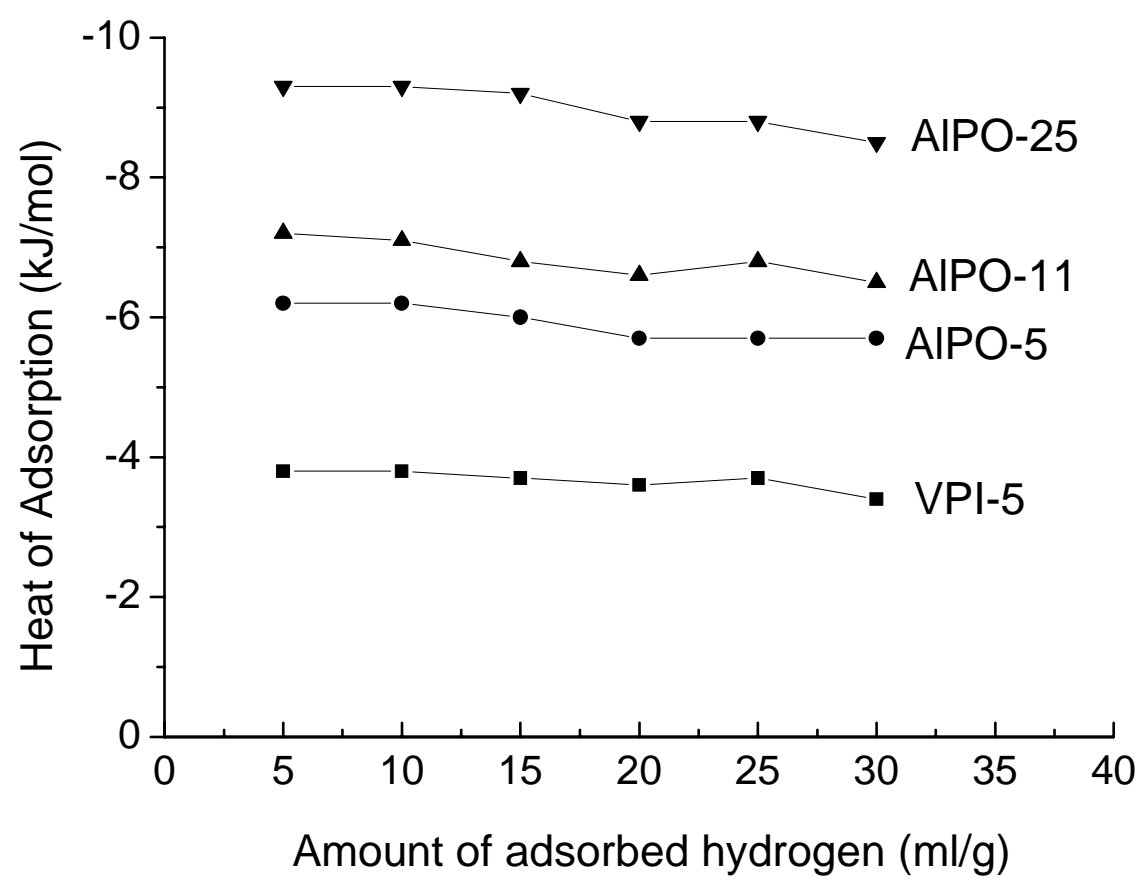

Supporting Figure 4. Heat of adsorption on various AlPOs. 